Editora Omnis Scientia

ANAIS DA I JORNADA NORTE-NORDESTE DE ENGENHARIAS (ONLINE)

Volume 1

$1^{\text {a }}$ Edição

TRIUNFO - PE 


\section{Coordenador Científico}

Alex Aguiar da Silva

\section{Coordenador de Publicação}

Daniel Luís Viana Cruz

\section{Coordenadora do Evento}

Andréa Telino Gomes

\section{Organizadores}

Academics - Eventos acadêmicos online

Editora Omnis Scientia

Andréa Telino Gomes

Daniel Luís Viana Cruz

Alex Aguiar da Silva

\section{Palestrantes}

Alex Aguiar da Silva

André Búrigo Leite

Cecília Aguiar da Silva

Eugênio Bastos da Costa

Jorge Recarte Henriquez Guerrero

Renan Ferreira da Rocha

\section{Avaliadores}

Alex Aguiar da Silva

Helio Cardoso Martim

Péricles Felipe Bastos Gomes

\section{Imagem de Capa}

Freepik

\section{Revisão}

Os autores

\section{(9) $\mathbb{\oplus \Theta \Theta}$}

Este trabalho está licenciado com uma Licença Creative Commons - Atribuição-NãoComercialSemDerivações 4.0 Internacional.

O conteúdo abordado nos artigos, seus dados em sua forma, correção e confiabilidade são de responsabilidade exclusiva dos autores. 


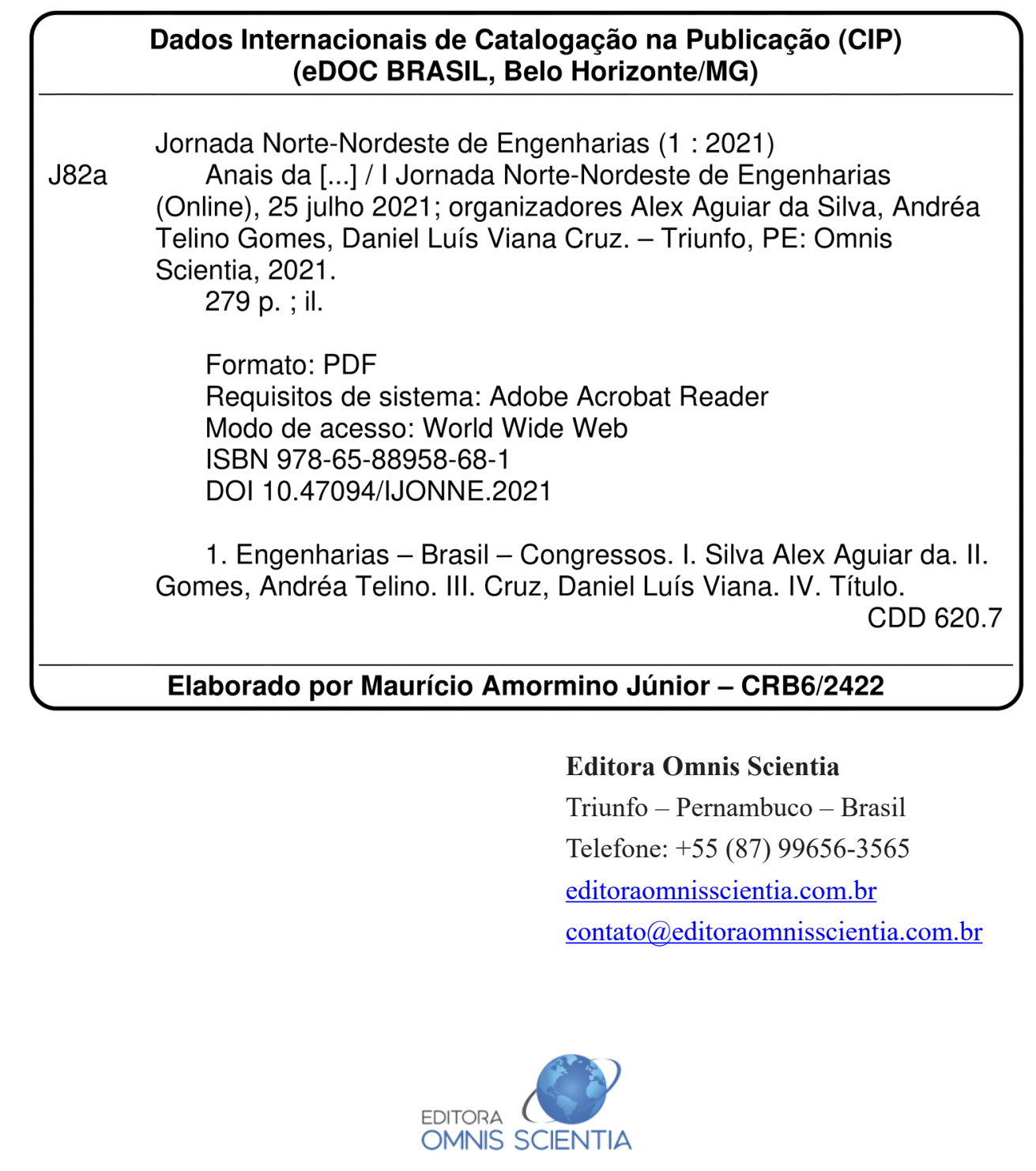




\section{EDITORIAL}

A grande área das engenharias abrangente uma série de ramos específicos, cada qual com determinados campos de aplicação e tipos de tecnologias, conjugando conhecimentos especializados no sentido de viabilizar as utilidades, tendo em conta a sociedade, a técnica, a economia e o meio ambiente. Deste modo, as engenharias aplicam o conhecimento científico, econômico, social e prático, para criar, desenhar, construir, manter e melhorar estruturas, máquinas, aparelhos, sistemas, materiais e processos. Assim, se adquire e se aplicam os conhecimentos matemáticos e técnicos na invenção, aperfeiçoamento e implementação de utilidades que realizem uma função ou objetivo.

A I Jornada Norte-Nordeste de Engenharias (online) I JONNE, objetivou reunir e integrar as engenharias para promover e apoiar esta área do conhecimento por meio de palestras, submissões de trabalhos na modalidade de resumos expandidos e exposição dos resumos aprovados.

Os participantes receberam certificados de participação de 20 horas e foi concedido menção honrosa para os três melhores trabalhos. Seguem os títulos dos resumos que foram premiados:

Modalidade resumo expandido

387210 - DIMENSIONAMENTO DE TROCADOR DE CALOR A PLACAS PARA PASTEURIZAÇÃO DE LEITE PELO PROCESSO HTST

387222 - DESENVOLVIMENTO DE UM DISPENSER DE ÁLCOOL GEL AUTOMÁTICO PARA MONITORAMENTO DA COMPLACÊNCIA DE MÃOS

387225 - REDE NEURAL ARTIFICIAL PARA VISCOSIDADE E CONDUTIVIDADE TÉRMICA DO FLUIDO REFRIGERANTE R32

A equipe organizadora da I JONNE agradece a todos os participantes, palestrantes e avaliadores pela participação e colaboração no congresso. 


\section{SUMÁRIO}

\section{ENGENHARIA CIVIL}

ANÁLISE DO USO DE RESÍDUO DE BRITAGEM DAS ROCHAS EM CONCRETO AUTOADENSÁVEL: UMA REVISÃO DA LITERATURA.....................................................12

ESTRATÉGIAS PARA A PREVENÇÃO DE ACIDENTES NA CONSTRUÇÃO CIVIL: UMA REVISÃO SISTEMÁTICA DA LITERATURA.

RESISTÊNCIA À COMPRESSÃO DE MISTURAS DE SOLO-CIMENTO COM NANOSSÍLICA E SÍLICA ATIVA.

PATOLOGIAS EM OBRAS PARALISADAS: O CASO DO ESTÁDIO COLOSSO DO TAPAJÓS NA CIDADE DE SANTARÉM - PA.

DIFERENÇA NO CUSTO DE MÃO DE OBRA EM FABRICAÇÃO DE LAJES PRÉ- MOLDADAS EM AMBITO NACIONAL

ESTUDO COMPARATIVO DE FUNDAÇÕES PARA UM EDIFÍCIO MODELO EM JOÃO PESSOA/PB: SAPATA X ESTACA HÉLICE CONTÍNUA.

MANIFESTAÇÕES PATOLÓGICAS EM EDIFICAÇÕES COM PAREDES DE CONCRETO ARMADO: ESTUDO DE CASO EM OBRA NA CIDADE DE JOÃO PESSOA - PB.....

REUTILIZAÇÃO DO PÓ RESIDUAL DE MARMORARIA PARA SUBSTITUIÇÃO DO AGREGADO MIÚDO NO CONCRETO.

SUBSIDÊNCIA OCORRIDA NOS BAIRROS PINHEIRO, BEBEDOURO E MUTANGE EM MACEIÓ - AL: UMA REVISÃO BIBLIOGRÁFICA.

CARACTERIZAÇÃO FÍSICA DAS VARIAÇÕES DIMENSIONAIS DO BAMBU GUADUA WEBERBAUERI DE RIO BRANCO - AC.

ESTUDO DA OCORRÊNCIA DE SOLOS COLAPSÍVEIS NA REGIÃO NORDESTE DO BRASIL.

OS EFEITOS DO USO DE POÇOS ARTESIANOS NO PROCESSO DE SALINIZAÇÃO COSTEIRA NA CIDADE DE SÃO LUÍS: UM LEVANTAMENTO BIBLIOGRAFICO.

TEORIADOS SÓLIDOS CELULARES EMPREGADOS EM REFORÇOS PARAACONSTRUÇÃO CIVIL

AVALIAÇÃO DO EXTRATO DE BOLDO E AROEIRA COMO INIBIDOR VEGETAL DE BAIXO CUSTO......

ANÁLISE DE DOSAGEM DE MATRIZ CIMENTÍCIA COM FIBRAS DE POLIPROPILENO: REVISÃO DA LITERATURA 
ANÁLISE DA RETENÇÃO DE CLORETOS EM ARGAMASSAS COM RESÍDUOS DE CELULOSE.

\section{ENGENHARIA DE MATERIAIS E METALÚRGICA}

ANÁLISE DA DEGRADAÇÃO FERRÍTICA DO AÇO INOXIDÁVEL DUPLEX SAF 2205 SUBMETIDO A ENVELHECIMENTO ISOTÉRMICO.

ANÁLISE DE INCLUSÕES E QUANTIFICAÇÃO DE POROSIDADE DO BRONZE AO ESTANHO EM ESTRUTURA BRUTA DE FUSÃO. 100

SÍNTESE RÁPIDA DE NANOFIOS DE NIOBATO DE SÓDIO. 105

AVALIAÇÃODAINCORPORAÇÃODACAREPADELAMINAÇÃOCOMOPARTEDAMATÉRIAPRIMA NA PRODUÇÃO DE SÍNTER DE FINOS DO MINÉRIO DE FERRO. 110

ESTUDO PROSPECTIVO DO PETRÓLEO APLICADO EM NANOMATERIAL CERÂMICO...... 115

ESTUDO PROSPECTIVO DE NANOMATERIAL CERÂMICO APLICADO EM SAÚDE MÉDICA E DENTÁRIA.

ESTUDO PROSPECTIVO DE MAGNETITA NANOMÉTRICA COMO MATERIAL FERROMAGNETICO.

ESTUDO PROSPECTIVO DE CERÂMICA AVANÇADA UTILIZANDO DOPAGEM......

BUSCA DE ANTERIORIDADE DE CERÂMICA APLICADA NA REMOÇÃO DE CONTAMINANTE..... .131

APLICAÇÕES DE $\mathrm{MoO}_{3}$ OBTIVO ATRAVÉS DE PRECIPITAÇÃO. 136

REVISÃO BIBLIOGRÁFICA SOBRE TUNGSTATO DE BÁRIO EM MEIO AQUOSO. 140

ESTUDOS DA DIFUSÃO DE CARBONO NOS AÇOS SAE 1020 E 1045 SUBMETIDOS À TRATAMENTO TERMOQUÍMICO DE CEMENTAÇÃO SÓLIDA. 145

\section{ENGENHARIA DO PRODUTO}

DESENVOLVIMENTO DE UM DISPENSER DE ÁLCOOL GEL AUTOMÁTICO PARA MONITORAMENTO DA COMPLACÊNCIA DE MÃOS. 150

\section{ENGENHARIA ELÉTRICA}

ENERGIA EÓLICA NO CEARÁ .156

\section{ENGENHARIA HIDRÁULICA}

ESTUDO DE MÉTODOS ANALÍTICOS PARA DETERMINAÇÃO DE PERDA DE CARGA EM TUBULAÇÕES DE PVC UTILIZADAS EM IRRIGAÇÕES. 162 
DESENVOLVIMENTO DE PRÁTICAS LABORATORIAIS APLICANDO OS CONCEITOS DE FENÔMENOS DE TRANSPORTES. 168

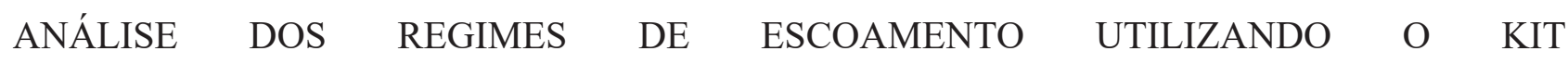
AQUALIBRIUM...

\section{ENGENHARIA MECÂNICA}

AVANÇO NO EMPREGO DE GABARITOS DE SOLDAGEM NO PROCESSO DE FABRICAÇÃO DE CHASSIS FORMULA SAE..

ANÁLISE DA DISTRIBUIÇÃO DE TEMPERATURA NOS PNEUS DE UM VEÍCULO FORMULA SAE PARA DIFERENTES CARGAS VERTICAIS. 184

\section{ENGENHARIA QUÍMICA}

UTILIZAÇÃO DE OXIDAÇÃO AVANÇADA PARA O TRATAMENTO DE EFLUENTES INDUSTRIAIS: UMA REVISÃO DA LITERATURA..

ANÁLISE DOS PRINCIPAIS PROCESSOS DE PRODUÇÃO DE BIODIESEL: UMA REVISÃO DE LITERATURA.

A QUÍMICA DOS AGROTÓXICOS USADOS EM AGRICULTURA: UMA REVISÃO DA LITERATURA. 201

AVALIAÇÃO CINÉTICA DA REAÇÃO DE DEGRADAÇÃO DE DIURON PRESENTE EM ÁGUAS SUPERFICIAIS.

ESTUDO DE CASO DO TRATAMENTO DE GÁS ÁCIDO COM COLUNA DE ABSORÇÃO DE LEITO RECHEADO.

ELABORAÇÃO DA CURVA DE CALIBRAÇÃO PARA ACRILAMIDA PELO MÉTODO DE CROMATOGRAFIA LÍQUIDA DE ALTA EFICIÊNCIA.

DETERMINAÇÃODECONSTANTESCINÉTICASPELOS MÉTODOSINTEGRALEDERUNGEKUTTA PARA REAÇÃO DE OXIDAÇÃO DO ANTRACENO.

DIMENSIONAMENTO DE TROCADOR DE CALOR A PLACAS PARA PASTEURIZAÇÃO DE LEITE PELO PROCESSO HTST.. 229

REDE NEURAL ARTIFICIAL PARA VISCOSIDADE E CONDUTIVIDADE TÉRMICA DO FLUIDO REFRIGERANTE R32.

TRANSFORMAÇÃO DO POLITEREFTALATO DE ETILENO RECICLADO EM FIBRAS TÊXTEIS DE POLIÉSTER. .239

\section{ENGENHARIA SANITÁRIA}

O USO DA ENERGIA SOLAR EM HOSPITAIS EM TEMPOS DE PANDEMIA 
ESTUDO DE VARIÁVEIS QUE POSSAM INFLUENCIAR NO CONSUMO DE ÁGUA DA CIDADE DE BAIXO GUANDÚ-ES........

\section{OUTRAS}

SISTEMAAUTOMÁTICO DE IRRIGAÇÃO. .260

A FRUTA DO MILAGRE - SYNSEPALUM DULCIFICUM.. 266 FOSSAS DE EVAPOTRANSPIRAÇÃO: UMA SOLUÇÃO SUSTENTÁVEL PARA OS DEFICITS NO SANEAMENTO EM ÁREAS CARENTES. .271

PROJETO DE ROBÓTICA SOCIAL E EDUCACIONAL .276 


\title{
ANÁLISE DOS REGIMES DE ESCOAMENTO UTILIZANDO O KIT AQUALIBRIUM
}

\author{
Cataryne Florencio Cardoso'; Nathália Francisca de Sousa Farias²; Renata Shirley Andrade \\ Araújo $^{3}$; Alessandro de Araújo Bezerra ${ }^{4}$. \\ 50 Graduanda, Universidade Federal do Piauí (UFPI), Teresina, Piauí. \\ ${ }_{51} \quad$ Graduanda, Universidade Federal do Piauí (UFPI), Teresina, Piauí. \\ ${ }_{52} \quad$ Doutora, Universidade Federal do Piauí (UFPI), Teresina, Piauí. \\ ${ }_{53} \quad$ Doutor, Universidade Federal do Piauí (UFPI), Teresina, Piauí.
}

DOI: 10.47094/IJONNE.2021.1

\begin{abstract}
RESUMO
O movimento dos fluidos pode ser classificado em laminar, turbulento e de transição. Os escoamentos laminar e turbulento são o foco de estudo de diversas áreas da engenharia, enquanto o escoamento de transição, por possuir características que transitam entre os dois tipos de escoamento, evidencia maior grau de complexidade. Dessa forma, o presente trabalho relaciona o fator de atrito obtido experimentalmente com os diferentes tipos de escoamentos e suas respectivas formulações empíricas existentes na literatura. Foram realizadas diversas simulações utilizando piezômetros ao longo de uma tubulação adutora montada com o "kit aqualibrium". Constatou-se que os fatores de atrito obtidos experimentalmente resultaram em valores abaixo do esperado e que, quanto maior o número de Reynolds, os resultados experimentais no regime de transição se aproximavam mais da fórmula do regime laminar. Também se observou que o fator de atrito no regime de transição não depende apenas do número de Reynolds.
\end{abstract}

PALAVRAS-CHAVE: Regimes de escoamento. Fator de Atrito. Número de Reynolds. ÁREA TEMÁTICA: Engenharia Hidráulica.

\section{INTRODUÇÃO}

Segundo Porto (2006), a metodologia de análise dos fluidos, em especial, da água, fundamenta-se em subdividir os escoamentos em grupos que compartilham de características comuns, a fim de serem estudados por métodos próprios. Na hidráulica, os escoamentos podem ser classificados em diversas categorias, como, por exemplo, escoamento unidimensional, bidimensional, uniforme, variado, fluvial e torrencial. No que tange ao número de Reynolds, têm-se o escoamento laminar, turbulento e de transição. 
De acordo com Porto (2006) e Azevedo Netto (1998), o escoamento é considerado turbulento para valores de Reynolds acima de 4000, além de apresentar trajetórias irregulares. No escoamento laminar, Porto (2006) afirma que o fluido percorre trajetórias paralelas e bem definidas, e ocorre para Rey $<2300$. O escoamento de transição, como o próprio nome sugere, encontra-se numa faixa intermediária de número de Reynolds $(2300<$ Rey $<4000)$ e, por se encontrar numa zona de transição, não possui características tão bem definidas quanto o escoamento laminar e turbulento, sendo válido o estudo da aplicabilidade dos três tipos de escoamentos, principalmente do regime de transição, que possui poucas pesquisas na literatura.

Na hidráulica, é recorrente o cálculo da perda de carga para a realização de estudos acerca dos fluxos de água em tubulações. Dentre as diversas formulações contidas na literatura, a perda de carga pode ser obtida através da fórmula de Darcy-Weisbach, que envolve um parâmetro $f$ denominado fator de atrito, o qual pode variar de acordo com o material da tubulação e as características do escoamento.

O "kit aqualibrium" é um equipamento utilizado em uma competição de modelagem para redes de distribuição de água que surgiu através de um projeto estudantil idealizado pelos alunos da Universidade de Joanesburgo. O equipamento contém peças em miniatura que simulam tubulações, conexões e reservatórios reais, permitindo colocar em prática os mais diversos conceitos da engenharia hidráulica.

Em virtude disso, o presente trabalho tem como objetivo comparar of para os diferentes tipos de escoamentos, obtidos em experimentos utilizando o "kit aqualibrium", e relacioná-los com as formulações empíricas existentes na literatura, além de analisar o comportamento apresentado em cada regime.

\section{METODOLOGIA}

Com o auxílio das peças de um "kit aqualibrium”, foi possível simular uma tubulação adutora, formando o seguinte sistema: tubulação horizontal com diâmetros de $3 \mathrm{~mm}$ e $6 \mathrm{~mm}$, três tubos na vertical representando piezômetros e um reservatório elevado mantido à nível constante, garantindo o regime permanente e obedecendo as regras da Equação de Bernoulli, conforme ilustra a figura 01 .

Foram efetuados diversos testes englobando os três tipos de escoamento. Para os regimes laminar e de transição, foram utilizadas tubulações de $3 \mathrm{~mm}$. Para o regime turbulento, os experimentos foram efetuados em tubulações com diâmetro de $6 \mathrm{~mm}$.

O número de Reynolds pode ser obtido após a aferição da vazão do escoamento. A perda de carga dos trechos (1-2, 2-3 e 3-4) pode ser encontrada através da altura dos piezômetros aplicadas na Equação de Bernoulli. A partir desses dados, é possível encontrar o fator de atrito $f$ experimental 
para cada um dos trechos, através da equação de Darcy-Weisbach.

Figura 01: Tubulação montada com Aqualibrium

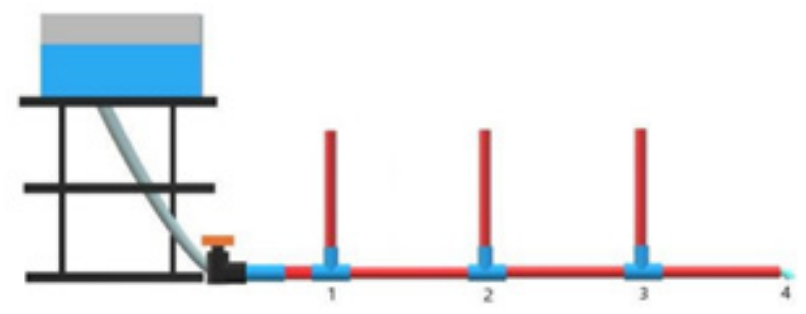

Fonte: Elaborada pelos autores (2021).

Também foram calculados os $f$ para os três tipos de regimes de escoamento. Para o escoamento de transição, utilizou-se a interpolação cúbica do ábaco de Moody. Para os escoamentos laminar, turbulento liso, turbulento rugoso e turbulento de transição, foram utilizadas, respectivamente, as equações $1,2,3$ e 4, onde $\varepsilon=0,00006 \mathrm{~m}$ é a rugosidade absoluta da tubulação e D é o diâmetro da tubulação (m).

$$
f=\frac{64}{R e y}
$$

$$
\frac{1}{\sqrt{f}}=2 \log \left(\frac{\operatorname{Rey} \sqrt{f}}{2,51}\right)
$$

$$
\frac{1}{\sqrt{f}}=2 \log \left(\frac{3,71 D}{\varepsilon}\right)
$$

(3)

$$
\frac{1}{\sqrt{f}}=-2 \log \left(\frac{\varepsilon}{3,71 D}+\frac{2,51}{\operatorname{Rey} \sqrt{f}}\right)
$$

\section{RESULTADOS E DISCUSSÕES}

A tabela 01 apresenta dados comparativos entre $f$ experimentais (trecho 1-2) e teóricos. 
Tabela 01: $f$ experimental versus $f$ matemático

\begin{tabular}{c|c|c|c|c|c|c|c|c|c|c}
\hline Rey & $\mathbf{f ( L a m i n a r )}$ & $\mathbf{f ( 1 - 2 )}$ & Rey & $\mathbf{f}($ Transição) & $\mathbf{f ( 1 - 2 )}$ & Rey & $\begin{array}{c}\text { f(Turb. f(Turb. de } \\
\text { Liso) }\end{array}$ & $\begin{array}{c}\mathbf{f}(\text { Turb. } \\
\text { Transicão) }\end{array}$ & Rugoso & $\mathbf{f ( 1 - 2 )}$ \\
\hline 1711,1 & 0,0374 & 0,0210 & $2.516,27$ & 0,0321 & 0,0241 & 5862,57 & 0,0357 & 0,0461 & 0,0379 & 0,0287 \\
\hline 1790,5 & 0,0357 & 0,0215 & $2.715,46$ & 0,0354 & 0,0101 & 7670,37 & 0,0332 & 0,0444 & 0,0379 & 0,0247 \\
\hline 1984,2 & 0,0323 & 0,0168 & $2.944,77$ & 0,0406 & 0,0146 & 9213,20 & 0,0316 & 0,0435 & 0,0379 & 0,0189 \\
\hline 2017,8 & 0,0317 & 0,0209 & $3.295,68$ & 0,0492 & 0,0158 & 10352,26 & 0,0306 & 0,0429 & 0,0379 & 0,0199 \\
\hline 2068,5 & 0,0309 & 0,0161 & $3.471,82$ & 0,0532 & 0,0124 & 11798,52 & 0,0296 & 0,0424 & 0,0379 & 0,0208 \\
\hline 2155,2 & 0,0297 & 0,0148 & $3.593,22$ & 0,0555 & 0,0139 & 12192,86 & 0,0293 & 0,0423 & 0,0379 & 0,0251 \\
\hline 2225,3 & 0,0288 & 0,0154 & $3.734,34$ & 0,0575 & 0,0169 & 13920,24 & 0,0283 & 0,0418 & 0,0379 & 0,0188 \\
\hline 2235,6 & 0,0286 & 0,0170 & $4.056,71$ & 0,0586 & 0,0143 & 14428,01 & 0,0281 & 0,0416 & 0,0379 & 0,0179 \\
\hline
\end{tabular}

Fonte: Elaborada pelos autores (2021).

Os fatores de atrito obtidos pelos experimentos com o "kit aqualibrium" (trechos 1-2, 2-3 e 3-4) resultaram em valores abaixo do esperado pelas formulações matemáticas. Para o escoamento laminar, isto é, Rey $<2300$, os valores de $f$ no trecho 1-2 diferiram em um erro médio de 43,88\% dos resultados fornecidos pela equação 1. Para o escoamento turbulento (Rey $>4000$ ), os resultados experimentais nos trechos 1-2 destoaram das equações 2, 3 e 4 com os respectivos erros: 31,33\%; $44,53 \%$ e $51,20 \%$. Para $2300<$ Rey $<4000$, ou seja, no escoamento de transição, a interpolação cúbica do ábaco de Moody resultou em um erro médio de $66,60 \%$, quando comparada com o fator de atrito experimental do trecho 1-2.

Para o regime de transição, constatou-se que, quanto maior o número de Reynolds, mais os valores experimentais de $f$ se aproximavam da fórmula do regime laminar, conforme apresenta o gráfico 01. Isso mostra que o fator de atrito no regime de transição não depende apenas do número de Reynolds, tendo em vista que os resultados de $f$, para números de Reynolds próximos de 4000, deveriam ser mais semelhantes aos $f$ para o escoamento turbulento e não o laminar.

Além disso, para o regime de transição, observou-se a ocorrência de oscilações na altura da lâmina d'água dos piezômetros, o que representa variações nas pressões dinâmicas ao longo do tempo. Percebe-se, ainda, que os valores de $f$ para os trechos 1-2, 2-3 e 3-4 diferem entre si. Essa diferença se dá devido às perdas de cargas localizadas, tendo em vista que as tubulações e conexões utilizadas no experimento possuem diâmetros bastante pequenos, o que intensifica a ocorrência de perdas localizadas. 
Gráfico 01: Relação entre fator de atrito e número de Reynolds

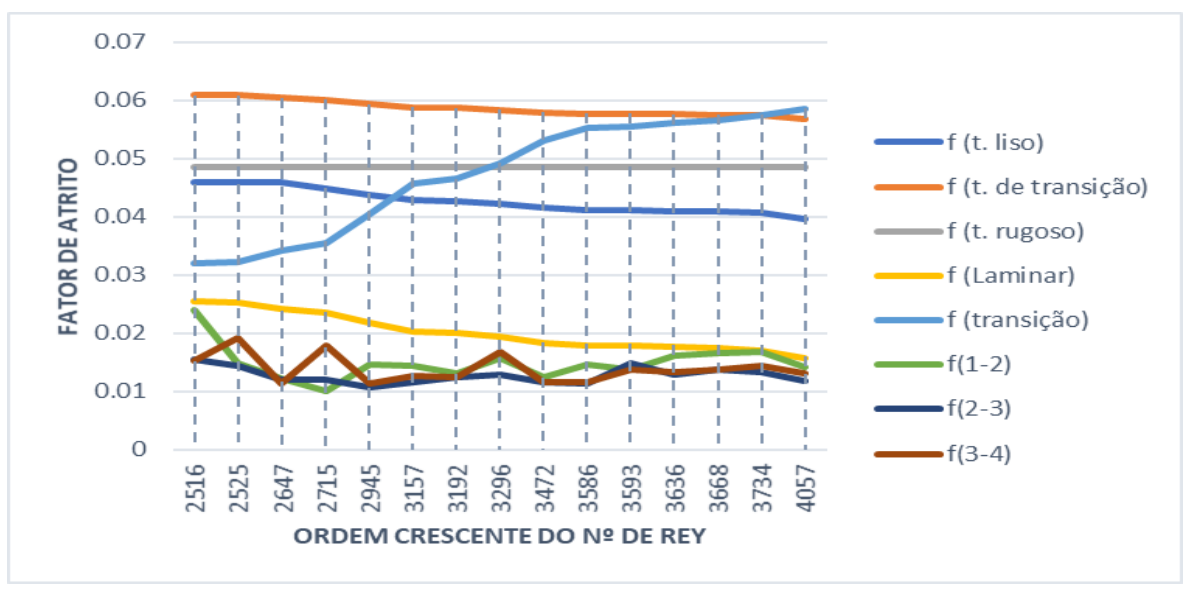

Fonte: Elaborado pelos autores (2021).

\section{CONSIDERAÇÕES FINAIS}

A partir dos resultados apresentados acima, é notória a existência de diferenças entre os valores de $f$ experimentais e calculados. Logo, tendo em vista que as tubulações utilizadas nos experimentos possuíam diâmetros extremamente pequenos, destaca-se a importância de desenvolver novos estudos com formulações envolvendo o fator de atrito que possam ser utilizadas nas circunstâncias apresentadas, visto que as fórmulas adotadas neste trabalho foram elaboradas para situações reais e tubulações maiores, o que possibilita a ocorrência de resultados conflitantes quando aplicadas em diâmetros menores.

\section{PRINCIPAIS REFERÊNCIAS}

PORTO, Rodrigo de Melo. Hidráulica Básica. 4. ed. São Carlos: EESC/USP, 2006. 519 p.

AZEVEDO NETTO, José Martiniano. Manual de Hidráulica. 8. ed. São Paulo: Blucher, $1998.669 \mathrm{p}$. 


\section{editoraomnisscientia@gmail.com M} https://editoraomnisscientia.com.br/

@editora_omnis_scientia @ https://www.facebook.com/omnis.scientia.9 f

$$
\text { +55 (87) 9656-3565 @ }
$$




\section{editoraomnisscientia@gmail.com M} https://editoraomnisscientia.com.br/ $\oplus$

@editora_omnis_scientia@ https://www.facebook.com/omnis.scientia.9 f

$$
\text { +55 (87) 9656-3565 @ }
$$

